${ }^{1}$ Unidad de Nefrología, Servicio de Medicina, Hospital Naval Almirante Nef. Viña del Mar, Chile.

${ }^{2}$ Departamento de Medicina, Escuela de Medicina, Universidad de Valparaíso. Viña del Mar, Chile.

${ }^{3}$ Departamento de Nefrología, Escuela de Medicina, Pontificia Universidad Católica de Chile. Santiago, Chile. ${ }^{4}$ Departamento de Endocrinología, Programa de Tumores Hipofisiarios, Pontificia Universidad Católica de Chile. Santiago, Chile.

Trabajo no recibió financiamiento. Los autores declaran no tener conflictos de interés.

Recibido el 29 de diciembre de 2019, aceptado el 18 de mayo de 2020 .

Correspondencia a: Jorge Vega Stieb. 5 Norte 1035, Viña del Mar Chile. jvegastieb@gmail.com

\section{Evaluación de la recolección de orina de 24 horas a partir de la creatininuria: fórmulas para estimarla y su rendimiento}

\author{
JORGE VEGA ${ }^{1,2}$, JUAN PABLO HUIDOBRO E. ${ }^{1,3}$, \\ FRANCISCO J. GUARDA ${ }^{4}$
}

\section{Performance of formulas for the evaluation of 24-hour urine collections using urine creatinine excretion}

Creatinine excretion is widely used as a method to evaluate the adequacy of urine collection in different clinical settings. Many factors influence its elimination, such as protein intake, exercise, muscle mass, age, and sex, among many others. As 24-hour urine collections can be cumbersome, several equations have been developed to aid clinicians to correctly interpret results derived from them. In this review article, we report the factors that can modify creatinine excretion and we evaluate the accuracy of different published equations to estimate 24hour urine creatinine excretion.

(Rev Med Chile 2021; 149: 242-247)

Key words: Creatinine; Clinical Laboratory Techniques; Data Collection; Urine.
L a medición de la excreción urinaria de creatinina en $24 \mathrm{~h}(\mathrm{UCr} / 24 \mathrm{~h})$ es utilizada frecuentemente por los médicos en la práctica clínica, para asegurar que la recolección de orina en $24 \mathrm{~h}$ ha sido completa e interpretar adecuadamente el clearance de creatinina. Para ello, multiplican la concentración de creatinina urinaria (expresada en $\mathrm{mg} / \mathrm{dl}$ ) por el número de decilitros de orina recolectados durante el período y el resultado expresado en $\mathrm{mg} / 24 \mathrm{~h}$ es dividido por el peso del paciente. Así se obtiene un valor de UCr en $\mathrm{mg} / \mathrm{kg} / 24 \mathrm{~h}$, el que se compara con los valores esperados en hombres o mujeres, según sea el caso. También se utiliza la UCr/24 h para la verificación de una recolección adecuada de orina en la medición de algunos analitos de interés clínico como proteínas y albúmina, electrolitos (sodio, potasio, cloro, fósforo, yodo), hormonas (aldosterona, cortisol, catecolaminas, metanefri- nas), metabolitos (ácido 5 hidroxi-indol acético) y drogas ilícitas. También se utiliza en el estudio metabólico en urolitiasis (calcio, ácido úrico, oxalatos, citratos, magnesio) y en la evaluación del balance nitrogenado (nitrógeno ureico urinario), entre muchos otros ${ }^{1-3}$.

La estimación de UCr/24 h puede ser útil para convertir la razón analito/creatinina de una muestra aislada de orina en una estimación de la excreción en 24 h del analito en cuestión ${ }^{2}$.

Actualmente, la recolección de orina de $24 \mathrm{~h}$ para la evaluación de la velocidad de filtración glomerular (VFG) ha sido reemplazada en la práctica clínica por ecuaciones basadas en la creatinina sérica y la detección de albuminuria y proteinuria en las nefropatías se realiza habitualmente a partir de índices en una muestra aislada en orina. Sin embargo, tanto para la medición de la proteinuria como para las otras mediciones antes menciona- 
das, la recolección de orina de 24 h sigue siendo el estándar ${ }^{3}$.

El gran problema que tiene la recolección del volumen de orina emitida en 24 h es la recolección incompleta, la que ocurre en alrededor de $30 \%$ de las recolecciones ${ }^{1}$. También ocurre en ocasiones en las que hay una sobre-recolección (cuando se incluye la primera orina de la mañana al inicio de la recolección). Estas dos situaciones modifican significativamente los resultados de las mediciones de los analitos investigados.

Por esta razón se han propuestos fórmulas para estimar la excreción de UCr/24 h las que permiten evitar los inconvenientes que tiene la recolección de orina de $24 \mathrm{~h}$ y considerar también los otros factores que influyen en la UCr/24 $\mathrm{h}^{1-8,11-13}$.

\section{Factores que influyen en la excreción de creatinina en orina}

La excreción de UCr varía entre rangos tan amplios como $<600$ y $>2.800 \mathrm{mg} /$ día. Ello se debe principalmente a diferencias en la masa muscular, ya que la creatinina proviene del catabolismo de la creatina y fosfocreatina, cuyas reservas están en el músculo esquelético. La cantidad de $\mathrm{UCr} / 24 \mathrm{~h}$ varía día a día, entre 3\% y 14\%. La afectan el ciclo menstrual y el stress emocional ${ }^{8}$. Influyen en forma importante la ingesta de proteínas y el ejercicio, que la aumentan ${ }^{1,3}$. La ingesta de carne de res y de pescado, que contienen creatinina, se suma a la creatinina endógena y aumenta su excreción renal $^{4,8,14}$.

La raza también influye. Los individuos sanos de raza negra excretan $5 \%$ más que los blancos, pareados por peso y edad ${ }^{4,6}$. Cuando están en hemodiálisis, los pacientes de raza negra tienen creatininemias superiores en $15 \%$ a $20 \%$ respecto a los pacientes blancos. Cuando se mide la creatinina extraída semanalmente en pacientes en diálisis, esta es $40 \%$ mayor respecto a sujetos de raza blanca, pareados por edad, peso y dosis de diálisis ${ }^{4}$.

La edad influye en forma importante en la UCr. A mayor edad, la eliminación de creatinina es menor ${ }^{6}$. En el estudio clásico que dio a conocer la fórmula de estimación del clearance de creatinina de Cockcroft-Gault, observaron que la UCr promedio descendía desde 23,6 a 12,1 $\mathrm{mg} / \mathrm{kg} /$ día, probablemente por la disminución de la masa muscular con la edad ${ }^{5,8}$. Existe un aumento en el porcentaje de grasa corporal con el paso del tiempo, así como una reducción de la masa muscular. Se ha estimado que corresponde a $2 \%$ por década ( $10 \%$ del peso corporal entre los 20 y 70 años) $)^{8}$. También se ha considerado la disminución en la ingesta de carne en las personas mayores, así como la disminución de la concentración de creatina (precursor de la creatinina) en los músculos ${ }^{8}$. El metabolismo de la creatinina y su excreción extrarrenal son menores en sujetos mayores, salvo en aquellos que tienen una función renal reducida ${ }^{8,10}$. Cantidades significativas de creatinina se eliminan por las heces y una parte de ella es degradada en el tubo digestivo ${ }^{15}$. En la insuficiencia renal crónica aumenta el clearance extrarrenal de creatinina y la excreción de creatinina por orina disminuye en hasta un tercio de la de un sujeto normal en pacientes con insuficiencia renal crónica avanzada ${ }^{8}$. Normalmente el clearance extrarrenal de creatinina es alrededor de $2 \%$ del clearance de creatinina ${ }^{8}$.

También Cockcroft y Gault observaron que la creatininuria diaria en las mujeres era menor que en los hombres y arbitrariamente la estimaron en alrededor de $85 \%$ de la eliminada por ellos ${ }^{5}$. Esto también se ha atribuido a su menor masa muscu$\operatorname{lar}^{10}$. Un estudio ajustado por el peso mostró que los hombres tuvieron una creatininuria diaria 33\% mayor que la de las mujeres ${ }^{10}$.

\section{Fórmulas para estimar la creatininuria}

Desde 1976 a la fecha se han publicado varias fórmulas para estimar la excreción urinaria de creatinina y así evitar los inconvenientes de la recolección de orina de $24 \mathrm{~h}$. En estas fórmulas se han incorporado variables demográficas como edad, sexo, raza, peso, superficie corporal y altu$\mathrm{ra}^{1,2,3,5,6,8,10,11}$. Como la mayoría de ellas han sido construidas con datos de pacientes con enfermedad renal crónica, su utilización en sujetos sanos o con masa muscular fuera de lo común no es recomendable ${ }^{3}$.

En 1976 Cockroft y Gault ${ }^{5}$ crearon una fórmula para estimar la creatininuria utilizando los datos de 249 hombres y la validaron en 236 pacientes de los cuales $96 \%$ eran hombres, la mayoría hospitalizados y con creatininemia estable, que tuvieron al menos 2 determinaciones de creatinina urinaria de $24 \mathrm{~h}$, que no difirieron en más de $20 \%$ entre 
ellas y que no fueron inferiores a $10 \mathrm{mg} / \mathrm{kg} /$ día. Las fórmulas desarrolladas fueron:

$$
\begin{gathered}
\text { Creatininuria en } 24 \mathrm{~h}(\mathrm{mg})=28-(0,2 \times \text { edad } \\
\text { en años) x peso }(\mathrm{kg}) \\
\text { (si es mujer multiplicar el resultado por } 0,85)
\end{gathered}
$$

\section{Hombres:}

$$
\begin{gathered}
\text { Creatininuria en } 24 \mathrm{~h}(\mathrm{mg} / \mathrm{kg})= \\
28-(0,2 \mathrm{x} \text { edad en años })
\end{gathered}
$$

Mujeres:

Creatininuria en $24 \mathrm{~h}(\mathrm{mg} / \mathrm{kg})=$ $28-(0,2 \times$ edad en años $) \times 0,85$

En un estudio de Jedrusik ${ }^{2}$ en que se evaluó el comportamiento de ocho fórmulas disponibles para estimar la $\mathrm{UCr} / 24 \mathrm{~h}$, la diferencia absoluta entre la creatininuria medida (mUCr/24 h) y la creatininuria estimada (eUCr/24 h) con la fórmula de Cockcroft-Gault fue $231 \mathrm{mg} / 24 \mathrm{~h}$ y la correlación (Pearson) entre ellas 0,79 cuando se utilizaron los criterios de inclusión propuestos por la Clínica Mayo (creatininuria medida de $13-29 \mathrm{mg} / \mathrm{kg} /$ día en hombres y $9-26 \mathrm{mg} / \mathrm{kg} /$ día en mujeres) ${ }^{16}$. El porcentaje de las estimaciones de $\mathrm{UCr} / 24 \mathrm{~h}$ que estuvieron dentro de $15 \%$ (P15) y de $30 \%(\mathrm{P} 30)$ de las creatininurias medidas fue $52 \%$ y $81 \%$, respectivamente. Cuando se usó un criterio de inclusión más estricto como el de Gerber y Mann ${ }^{1}$ (hombres: 15-25 mg/kg/día; mujeres: 10-20 mg/ $\mathrm{kg} /$ día) la correlación (Pearson) entre la eUCr/24 h y mUCr/24 h fue superior $(0,87)$ así como P15 y P30: $49 \%$ y $82 \%$, respectivamente (Tabla 1 ).

En 1984 Imbembo y Walser ${ }^{9}$ publicaron unas tablas en que expresaron los valores de $\mathrm{UCr} / 24 \mathrm{~h}$ esperados tanto en hombres como mujeres según su peso ideal, dependiendo de su altura y de su edad. Estas tablas fueron utilizadas 3 años más tarde por Walser para compararlas con las creatininurias medidas como una forma de evaluación de la nutrición proteica en adultos de diversas edades ${ }^{8}$.

En 1997 Goldwasser et $\mathrm{al}^{4}$, con el objeto de conocer si la raza o la presencia de diabetes mellitus tenían influencia en la $\mathrm{UCr} / 24 \mathrm{~h}$ en hombres con insuficiencia renal crónica moderada (clearance de creatinina $48 \pm 35 \mathrm{ml} / \mathrm{min}$ ), efectuaron un estudio en 2 series que reunieron a 101 pacientes $(35 \%$ de raza negra, $38 \%$ diabéticos). Encontraron que los pacientes de raza negra tenían una $\mathrm{UCr} / 24 \mathrm{~h}$ superior a los de raza blanca y que la presencia de diabetes no influía en la misma. Desarrollaron la siguiente fórmula para hombres de $\geq 50$ años:

$$
\begin{gathered}
\text { Creatininuria en } 24 \mathrm{~h}(\mathrm{mg})=23,57-\mathrm{edad} / 8,25 \\
+1,93(\text { si es de raza negra) } \mathrm{x} \text { peso }(\mathrm{kg})
\end{gathered}
$$

La correlación entre los valores medidos y los estimados con esta ecuación, así como los valores de P15 y P30 en los trabajos de Jedrusik ${ }^{2}$ e Ix y Levey $^{3}$ se muestran en la Tabla 1.

El 2009 Rule y $\mathrm{col}^{11}$ publicaron una fórmula para estimar el clearance de creatinina y $\mathrm{UCr} / 24 \mathrm{~h}$, la cual desarrollaron con los datos demográficos y la medición de la masa muscular en 664 miembros de la población de Minnesota quienes recolectaron ambulatoriamente la orina de $24 \mathrm{~h}$. De ellos, $51 \%$ fueron mujeres y su edad promedio $55+/-20$ años. Excluyeron a los individuos blancos de origen hispánico dado que la raza altera la relación entre la creatinina sérica y la VFG. Encontraron que las ecuaciones disponibles que usan datos demográficos para estimar la generación de creatinina sobreestiman el diagnóstico de enfermedad renal crónica en sujetos mayores y en mujeres y también sobreestiman el riesgo de sufrir eventos cardiovasculares.

La fórmula desarrollada fue:

Creatininuria en $24 \mathrm{~h}(\mathrm{mg})=$

$\{\operatorname{Exp}[7,26-0,26$ (si es mujer) $-(0,011 \mathrm{x}$

(edad - 55) si la edad > 55 años)] $\}$ x área de superficie corporal/1,73 $\left(\mathrm{m}^{2}\right)$

Los valores de P15, P30 y la correlación entre los valores medidos y estimados con esta fórmula en los trabajos de Jedrusik ${ }^{2}$ e Ix y Levey ${ }^{3}$ se muestran en la Tabla 1.

El 2011 Ix y Levey (del grupo que creó la fórmula para estimar la VFG "CKD-EPI" ${ }^{17}$ ) publicaron una investigación en la cual reunieron los datos de 6 estudios en que los participantes $(\mathrm{n}=3.453)$ recolectaron ambulatoriamente la orina de $24 \mathrm{~h}$. Incluyeron a hombres $(58,5 \%)$, mujeres $(41,5 \%)$ e individuos de raza negra $(8,8 \%)$, con edades promedio de 46 años. Con 3 estudios $(\mathrm{n}=2.466$ pacientes $)$ desarrollaron una fórmula de estimación de UCr/24 h y la validaron internamente. Para la validación externa utilizaron los otros tres estudios ( $\mathrm{n}=987$ pacientes). Excluyeron a los sujetos con $\mathrm{UCr} / 24 \mathrm{~h}<350$ o $>3.500 \mathrm{mg} /$ día por ser biológicamente implausibles. 
Tabla 1. Comportamiento de 8 ecuaciones disponibles para estimar la creatininuria de 24 horas utilizando datos demográficos, respecto a la creatininuria medida

\begin{tabular}{|c|c|c|c|c|c|}
\hline Ecuación & Evaluación & Criterios & Correlación & P15 (\%) & P30 (\%) \\
\hline \multirow[t]{3}{*}{ Cockroft y Gault ${ }^{5}$} & Jedrusik² & Clínica Mayo ${ }^{16}$ & 0,79 & 52 & 81 \\
\hline & Jedrusik² & Gerber y Mann¹ & 0,87 & 49 & 82 \\
\hline & Ix y Levey ${ }^{3}$ & & & 49 & 76 \\
\hline \multirow[t]{3}{*}{ Goldwasser et $\mathrm{al}^{4}$} & Jedrusik ${ }^{2}$ & Clínica Mayo ${ }^{16}$ & 0,72 & 42 & 75 \\
\hline & Jedrusik² & Gerber y Mann¹ & 0,84 & 53 & 92 \\
\hline & Ix y Levey ${ }^{3}$ & & & 45 & 76 \\
\hline \multirow[t]{3}{*}{ Ix y Levey ${ }^{3}$} & Jedrusik ${ }^{2}$ & Clínica Mayo ${ }^{16}$ & 0,83 & 54 & 86 \\
\hline & Jedrusik ${ }^{2}$ & Gerber y Mann¹ & 0,93 & 53 & 94 \\
\hline & Rule y col ${ }^{11}$ & & & 46 & 81 \\
\hline \multirow[t]{2}{*}{ Gerber y Mann ${ }^{1}$} & Jedrusik ${ }^{2}$ & Clínica Mayo ${ }^{16}$ & 0,81 & 33 & 56 \\
\hline & Jedrusik ${ }^{2}$ & Gerber y Mann ${ }^{1}$ & 0,93 & 74 & 99 \\
\hline \multirow[t]{2}{*}{ Kawasaki11 } & Jedrusik ${ }^{2}$ & Clínica Mayo ${ }^{16}$ & 0,84 & 53 & 80 \\
\hline & Jedrusik ${ }^{2}$ & Gerber y Mann¹ & 0,92 & 78 & 99 \\
\hline \multirow[t]{3}{*}{ Walser $^{8}$} & Jedrusik ${ }^{2}$ & Clínica Mayo ${ }^{16}$ & 0,81 & 56 & 82 \\
\hline & Jedrusik² & Gerber y Mann¹ & 0,90 & 62 & 97 \\
\hline & Ix y Levey ${ }^{3}$ & & & 47 & 78 \\
\hline \multirow[t]{3}{*}{ Rule $^{7}$} & Jedrusik ${ }^{2}$ & Clínica Mayo ${ }^{16}$ & 0,83 & 56 & 89 \\
\hline & Jedrusik ${ }^{2}$ & Gerber y Mann¹ & 0,92 & 55 & 95 \\
\hline & Ix y Levey ${ }^{3}$ & & & 46 & 81 \\
\hline \multirow[t]{2}{*}{ Tanaka $^{12}$} & Jedrusik² & Clínica Mayo ${ }^{16}$ & 0,76 & 40 & 63 \\
\hline & Jedrusik ${ }^{2}$ & Gerber y Mann ${ }^{1}$ & 0,87 & 68 & 95 \\
\hline
\end{tabular}

Tabla adaptada de la publicada por Jedrusik et $a^{2}$. Criterio de aceptación de la recolección de orina $24 \mathrm{~h}$ de Clínica Mayo = Hombres: 13-29 mg/kg/día; Mujeres: 9-26 mg/kg/día. Criterio de aceptación de la recolección de orina 24 h de Gerber y Mann = Hombres: $\geq 20$ mg/kg/día; Mujeres: $\geq 15$ mg/kg/día. Correlación: Correlación de Pearson entre la creatininuria medida y estimada en 24 h. P15: Proporción de creatininurias estimadas con la ecuación que estuvieron dentro de un 15\% de las creatininurias medidas en orina de 24 h. P30: Proporción de creatininurias estimadas con la ecuación que estuvieron dentro de un $30 \%$ de las creatininurias medidas en orina de $24 \mathrm{~h}$.

La fórmula desarrollada fue:

Creatininuria en $24 \mathrm{~h}(\mathrm{mg})=879,89+12,51 \mathrm{x}$ peso $(\mathrm{kg})-6,19 \mathrm{x}$ edad (años) $+34,51$ (si es de raza negra) $-379,42$ (si es mujer)

Los porcentajes de las eUCr/24 h con esta fórmula que estuvieron dentro de $15 \%$ (P15) y de $30 \%$ (P30) de las mUCr/24 h fue 59\% y $85 \%$ en el grupo de validación interna y de 51\% y $79 \%$ en el grupo de validación externa. Los porcentajes obtenidos al emplear las ecuaciones de Rule, Walser, Cockcroft-Gault y Goldwasser ${ }^{4,5,8,11}$ se muestran en la Tabla 1.

El 2014 Gerber y Mann ${ }^{1}$ publicaron otra fórmula para estimar UCr/24 h. Reclutaron indivi- duos mayores de 21 años incluyendo hipertensos y sujetos con disfunción renal, los que recolectaron orina de $24 \mathrm{~h}$ ingiriendo 3 litros de jugo durante el período. Consideraron incompletas a las recolecciones cuando las creatininurias fueron $<20$ $\mathrm{mg} / \mathrm{kg}$ en hombres y $<15 \mathrm{mg} / \mathrm{kg}$ en mujeres. Para el desarrollo de la fórmula incluyeron a 50 individuos con recolecciones completas y luego la validaron en otra cohorte de 83 pacientes.

La fórmula desarrollada fue:

Creatininuria en $24 \mathrm{~h}(\mathrm{mg})=699-421,9$

(si es mujer $)+(16,83 \times$ peso en $\mathrm{kg})-25,82$

(si es de raza blanca) - 2,67 x edad (años)

La correlación entre la mUCr/24 h y eUCr/24 h 
Tabla 2. Poblaciones en que fueron desarrolladas y validadas las ecuaciones disponibles para estimar la creatininuria de $\mathbf{2 4}$ h utilizando datos demográficos

\begin{tabular}{|c|c|}
\hline Ecuación & Características de las poblaciones utilizadas \\
\hline Cockroft y Gault ${ }^{5}$ & $\begin{array}{l}\text { Desarrollada y validada internamente en } 505 \text { pacientes, la mayoría internados en hospitales canadien- } \\
\text { ses, } 96 \% \text { fueron hombres. Las edades variaron entre } 18 \text { y } 92 \text { años }\end{array}$ \\
\hline Goldwasser et al ${ }^{4}$ & $\begin{array}{l}\text { Desarrollada en } 35 \text { hombres y validada internamente en } 66 \text { hombres norteamericanos, que incluyó } \\
\text { en más del } 90 \% \text { a sujetos de raza blanca o negra y a escasos de origen hispánico o asiáticos. La edad } \\
\text { promedio fue } 66 \text { años. Alrededor de un } 45 \% \text { eran diabéticos }\end{array}$ \\
\hline Ix y Levey 3 & $\begin{array}{l}\text { Desarrollada en } 1.644 \text { pacientes pertenecientes a } 26 \text { estudios hechos previamente. Fue validada inter- } \\
\text { namente en } 822 \text { y externamente en } 987 \text {. Fueron hombres el } 60 \% \text {, de raza blanca } 90 \% \text { y de raza negra } \\
10 \% \text {. La edad promedio fue } 46 \text { años }\end{array}$ \\
\hline Rule et $\mathrm{al}^{7,10}$ & $\begin{array}{l}\text { Desarrollada en } 664 \text { pacientes ambulatorios de raza blanca, de Rochester, Minnesota, USA. El 51\% eran } \\
\text { mujeres y su edad } 55 \pm 20 \text { años. Se excluyó a los pacientes blancos de origen hispánico }\end{array}$ \\
\hline Kawasaki ${ }^{11,18}$ & $\begin{array}{l}\text { Desarrollada en } 247 \text { sujetos sanos de origen japonés ( } 47 \% \text { mujeres) con edades entre } 20 \text { y } 84 \text { años. } \\
\text { Fue validada en } 85 \text { sujetos sanos de origen japones, nepalí, norteamericanos e italianos }\end{array}$ \\
\hline Tanaka12 & Desarrollada en 591 japoneses sanos provenientes de 3 ciudades con edades promedio de $40 \pm 11$ años \\
\hline Walser ${ }^{8,9}$ & $\begin{array}{l}\text { Construyeron un nomograma que incluyó sexo, peso y altura utilizando los datos de } 5 \text { estudios efec- } \\
\text { tuados en sujetos sanos de la comunidad u hospitalizados, norteamericanos, libres de enfermedad } \\
\text { renal o hepática }\end{array}$ \\
\hline Gerber $^{1}$ & $\begin{array}{l}\text { Fue desarrollada en } 50 \text { pacientes norteamericanos sanos o hipertensos ( } 74 \% \text { ) con edades promedio } \\
50 \pm 15 \text { años, } 50 \% \text { mujeres, } 60 \% \text { de raza blanca y } 40 \% \text { de raza negra. Fue validada en } 83 \text { sujetos } \\
\text { mayoritariamente blancos ( } 89 \%) \text {, hipertensos ( } 87 \% \text { y con edades promedio } 59 \pm 12 \text { años }\end{array}$ \\
\hline
\end{tabular}

fue 0,80 en la cohorte completa y 0,93 en los que tuvieron recolecciones completas. Los valores obtenidos en el trabajo de Jedrusik ${ }^{2}$ se muestran en la Tabla 1.

Existen otras 2 ecuaciones propuestas por los autores asiáticos Tanaka y Kawasaki para estimar la UCr/24 h que se han incluido dentro de ecuaciones para predecir la excreción urinaria de sodio y potasio en $24 \mathrm{~h}$ con muestras aisladas de orina ${ }^{11-13}$.

\section{Ecuación de Tanaka}

Creatininuria en $24 \mathrm{~h}(\mathrm{mg})=-2,04 \mathrm{x}$ edad (años) $+14,89 \times$ peso $(\mathrm{kg})+16,14 \mathrm{x}$ altura $(\mathrm{cm})$

\section{Ecuación de Kawasaki} $-2244,45$

Creatininuria en $24 \mathrm{~h}(\mathrm{mg})=-4,72 \mathrm{x}$ edad $($ años $)+8,58 \times$ peso $(\mathrm{kg})+5,09 \times$ altura $(\mathrm{cm})-$ 74,5 (en hombres).

Creatininuria en $24 \mathrm{~h}(\mathrm{mg})=-12,63 \times$ edad $($ años $)+15,12 \times$ peso $(\mathrm{kg})+7,39 \times$ altura $(\mathrm{cm})$

$$
\begin{gathered}
-79,9 \text { (en mujeres). } \\
(\mathrm{cm})-2244,45 \text {. }
\end{gathered}
$$

Los valores de P15, P30 y la correlación entre los valores medidos y estimados con estas dos ecuaciones publicados en el trabajo de Jedrusik ${ }^{2}$ se muestran en la Tabla 1.

En suma: Existen actualmente 8 ecuaciones para estimar la excreción urinaria de creatinina de $24 \mathrm{~h}$. Estas ecuaciones son útiles para comparar las creatininurias estimadas con la medidas en una recolección de orina de 24 h, lo que permite evaluar si una recolección es correcta para su análisis, especialmente en pacientes en que el diagnóstico depende de la correcta interpretación de los exámenes de orina, como en la evaluación de la función renal, balance nitrogenado, síndrome de Cushing, hiperaldosteronismo primario, feocromocitoma, síndrome carcinoide, estudio de urolitiasis recurrente, reabsorción tubular de fósforo en hipofosfemias, yoduria, etc. También sirven para estimar la creatininuria prescindiendo de una recolección de orina de $24 \mathrm{~h}$ en la estimación de la excreción de sodio o potasio en una muestra aislada de orina en la evaluación de pacientes hipertensos ${ }^{11-13}$. 
La correlación de Pearson entre la creatininuria medida y la creatininuria estimada varía entre 0,72 y 0,84 entre las 8 ecuaciones, cuando se utilizan los criterios de inclusión para aceptar las recolecciones de orina de 24 h de la Clínica Mayo. Cuando se emplea un criterio más estricto para aceptar una recolección como el de Gerber y Mann, la correlación de Pearson mejora a 0,84 y 0,93.

Cuando se acepta que los valores de la creatininuria estimada estén dentro de un rango no mayor de $30 \%$ de la creatininuria medida, las ecuaciones de Rule y CKD-EPI ${ }^{3,11}$ tuvieron un mejor comportamiento que las restantes; en cambio, cuando se utilizaron criterios más estrictos como el de Gerber y Mann, todas las ecuaciones salvo la de Cockcroft-Gault estuvieron dentro de ese rango en más de $90 \%$.

Con la información disponible pueden utilizarse para los fines mencionados cualquiera de las ecuaciones existentes (salvo la de Cockcroft-Gault) cuando se utilizan criterios estrictos para aceptar como útil una recolección de orina de $24 \mathrm{~h}$. Cuando se utilizan criterios más laxos deben preferirse las ecuaciones de Rule y CKD-EPI ${ }^{3,11}$.

\section{Referencias}

1. Gerber LM, Mann SJ. Development of a model to estimate 24-hour urinary creatinine excretion. J Clin Hypertens (Greenwich) 2014; 16: 367-71.

2. Jedrusik P, Symonides B, Gaciong Z. Performance of 24-hour urinary creatinine excretion-estimating equations in relation to measured 24-hour urinary creatinine excretion in hospitalized hypertensive patients. Sci Rep. 2019; 9: 3593.

3. Ix JH, Wassel CL, Stevens LA, Beck GJ, Froissart M, Navis G, et al. Equations to estimate creatinine excretion rate: the CKD epidemiology collaboration. Clin J Am Soc Nephrol. 2011; 6: 184-91.

4. Goldwasser P, Aboul-Magd A, Mahendra M. Race and creatinine excretion in chronic renal insufficiency. Am J Kidney Dis. 1997; 30: 16-22.

5. Cockcroft DW, Gault MH. Prediction of creatinine clearance from serum creatinine. Nephron. 1976; 16: 31-41.

6. James GD, Sealey JE, Alderman M, Ljungman S, Mueller FB, Pecker MS, et al. A longitudinal study of urinary creatinine and creatinine clearance in normal subjects. Race, sex, and age differences. AJH 1988; 1: 124-131.
7. Rule AD, Bailey KR, Schwartz GL, Khosla S, Lieske JC, Melton III LJ. For estimating creatinine clearance measuring muscle mass gives better results tan those base don demographics. Kidney Int. 2009; 75: 1071-8.

8. Walser M. Creatinine excretion as a measure of protein nutrition in adults of varying age. J Parenter Enteral Nutr. 1987; 11: 73S.

9. Imbembo AL, Walser M. Nutritional assessment. In Nutritional assessment, the John Hopkins handbook (eds Walser, M., Imbembo, A.L. Y Margolis, S) 9-30 (W.B.Saunders, 1984).

10. Rule AD, Larson TS, Bergstralh EJ, Slezac JM, Jacobsen SJ, Cosio GF. Using serum creatinine to estimate glomerular filtration rate: accuracy in Good health and chronic kidney disease. Ann Intern Med. 2004; 141: 92937.

11. Kawasaki T, Itoh K, Uezono K, Sasaki H. A simple method for estimating $24 \mathrm{~h}$ urinary sodium and potassium excretion from second morning voiding urine specimen in adults. Clin Exp Pharmacol Physiol. 1993; 20: 7-14.

12. Tanaka T, Okamura T, Miuria K, Kadowaki T, Ueshima $\mathrm{H}$, Nakawawa $\mathrm{H}$, et al. A simple method to estimate populational 24-h urinary sodium and potassium excretion using a casual urine specimen. J Hum Hypertens 2002; 16: 97-103.

13. Mente A, O’Donnell MJ, Dagenais G, Wielgosz A, Lear SA, McQueen MJ, et al. Validation and comparison of three formulae to estimate sodium and potassium excretion fron a single morning fasting urine compared to 24-h measures in 11 countries. J Hypertens 2014; 32; 1005-15.

14. Vega J, Huidobro JP. Reserva funcional renal. Concepto y aplicabilidad potencial en la práctica clínica. Rev Med Chile 2019; 147: 1323-8.

15. Vega J, Huidobro JP. Efectos en la función renal de la suplementación de creatina con fines deportivos. Rev Med Chile 2019; 147: 628-33.

16. Mayo Clinic, Mayo Medical Laboratories. Creatinine, 24 hour, urine, https://www.mayomedicallaboratories.com/ test-catalog/Clinical+and+Interpretative/8513(2018).

17. Levey AS, Stevens LA, Schmid CH, Zhang YL, Castro AF 3rd, Feldman HI, et al. A new equation to estimate glomerular filtration rate. Ann Intern Med. 2009; 150: 604-12.

18. Kawasaki T, Uezono K, Itoh K, Ueno M. Prediction of 24-hour urinary clearance excretion from age, body weight and height of an individual and its application. Japanese Journal of Public Health. 1991; 38: 567-74. 